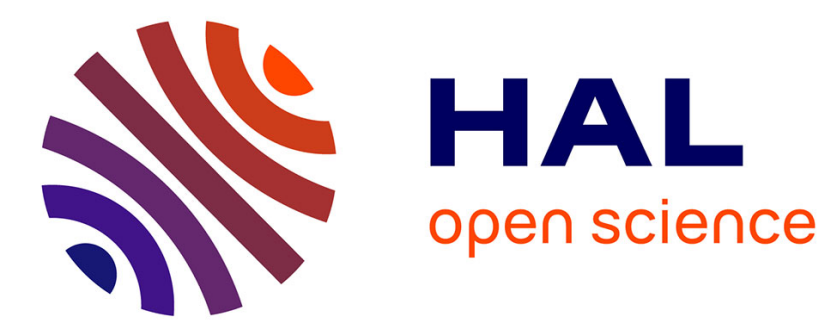

\title{
RECENT ADVANCES IN WAVES IN LAYERED MEDIA
}

\author{
S. Rokhlin
}

\section{To cite this version:}

S. Rokhlin. RECENT ADVANCES IN WAVES IN LAYERED MEDIA. Journal de Physique IV Proceedings, 1992, 02 (C1), pp.C1-819-C1-826. 10.1051/jp4:19921179 . jpa-00251140

\section{HAL Id: jpa-00251140 https://hal.science/jpa-00251140}

Submitted on 1 Jan 1992

HAL is a multi-disciplinary open access archive for the deposit and dissemination of scientific research documents, whether they are published or not. The documents may come from teaching and research institutions in France or abroad, or from public or private research centers.
L'archive ouverte pluridisciplinaire HAL, est destinée au dépôt et à la diffusion de documents scientifiques de niveau recherche, publiés ou non, émanant des établissements d'enseignement et de recherche français ou étrangers, des laboratoires publics ou privés. 


\title{
RECENT ADVANCES IN WAVES IN LAYERED MEDIA
}

\author{
S.I. ROKHLIN \\ Department of Welding Engineering, The Ohio State University, 190 West 19th Avenue, Columbus, \\ Ohio 43210, USA
}

\begin{abstract}
The topic of wave propagation in layered media has for a long time been of major interest in underwater and geophysical acoustics. Later on interest in this field arose due to needs in acousto-microelectronics, mainly surface acoustic wave devices, nondestructive evaluation of composite and layered materials and oil exploration. New applications have presented new challenging theoretical and experimental problems. One trend is to describe more complicated media including anisotropy, nonhomogeneity, imperfect interfaces, and wave diffraction on inhomogeneities; a second trend is related to solutions of the inverse problem, in which properties of the media should be determined from the wave signature. In this paper we review recent developments in theoretical and experimental studies of elastic wave propagation in layered media with applications to ultrasonic evaluation of materials. We will focus mainly on anisotropy and media property reconstruction. We will also discuss the boundary conditioned for the imperfect interface, which may involve partial contact or other interface inhomogeneities. Such generalized boundary conditions can be systematically incorporated in the transfer matrix approach.
\end{abstract}

La propagation d'ondes en milieu stratifié est depuis longtemps un sujet d'étude majeure dans les domaines d'acoustique sous-marine et géophysique. L'intérêt dans ce champs d'étude s'est depuis lors étendu aux domaines de l'acousto-microélectronique, principalement relié aux dispositifs générateur d'ondes acoustiques de surface, de l'évaluation non-destructive des materiaux composites et stratifiés, et de l'exploration des champs pétrolifères. Ces nouvelles applications ont introduit de nouveaux problèmes tant théorique qu'expérimentaux. Une solution possible est de décrire ce milieu complexe incluant anisotropie, non-homogénéité, interfaces imparfaites, et la diffraction d'ondes sur les inhomogénéités; une seconde solution est reliée aux solutions du problème inverse dans lequel les propriétés du milieu étudié sont déterminées à partir des données receuillies. Dans cette étude, nous revisons les plus récents développements expérimentaux et théoriques dans le domaine de la propagation d'ondes acoustiques en milieu stratifié appliqués à l'évaluation des matériaux. Le focus de notre étude porte sur la reconstruction des propriétés et de l'anisotropie du milieu. Nous allons de plus discuter les conditions de limite pour une interface imparfaite, ceci peut impliquer un contact partiel ou autre inhomogénéités à l'interface. Ces conditions de limite généralisées peuvent être incorporées systématiquement dans l'approche de matrice de transfert.

\section{Matrix approach}

To solve the problem of reflection and refraction on the interface between two solids, one must formulate a linear system of equations from the boundary conditions -4 in the isotropic case and 6 in the anisotropic. The number of these boundary conditions grows to 8 when one incorporates piezoelectricity. The direct approach to an $\mathrm{N}$-layered medium will lead to an $m \times N$ system of equations, where $m$ is the number of boundary condition equations for one interface. For a 
large system of layers, and for nonhomogeneous layers, solution becomes excessively tedious. A matrix approach which has significantly improved the treatment of this problem was suggested by Thompson [1] and later by Haskell [2]. The method is described in Brekhovskikh [3]. For a given layer of thickness $h$ they introduce boundary vectors on each surface of the layer

$$
U^{\prime}(0)=\left(u_{x}^{\prime}, u_{z}^{\prime}, \sigma_{z z}^{\prime}, \sigma_{z x}^{\prime}\right)^{T}, \quad U(h)=\left(u_{x}, u_{z}, \sigma_{z z}, \sigma_{z x}\right)^{T}
$$

and found a transfer matrix $B$ which relates the boundary vector from one surface of the layer to another.

$$
U(h)=B U^{\prime}(0)
$$

where $B$ is a $4 \times 4$ transfer matrix. Repeating this procedure recursively for each layer in the system one forms the transfer matrix for $N$-layered system

$$
B=\prod_{i=1}^{N} B_{i} \quad i=1, \cdots, 6
$$

which relates stresses and displacements on the input and output sides of the layered system. For reflection and transmission coefficients the problem becomes similar to that of a single interface where the matrix $B$ should be multiplied by the matrix semispaces $C$

$$
\left[\phi_{N}, R_{l}^{N}, \psi_{N}, R_{t}^{N}\right]^{T}=C^{N} B C^{0}\left[R_{l}^{0}, \phi_{0}, R_{t}^{0}, \psi_{0}\right]^{T}
$$

where $R_{l}, R_{t}$ are the reflection and transmission coefficients in semispaces $N$ and 0 and $\phi, \psi$ are the elastic potentials of the incident waves in these semispaces (if only one wave is incident, the other incident potentials should be set to zero).

Later it was found that the method became unstable when the layer thickness was comparable to the wavelength. This instability has been analyzed and ways to overcome it suggested by Dunkin [4] and Thrower [5]; see also [6]. This modification leads to a $6 \times 6$ transfer matrix, instead of a $4 \times 4$ transfer matrix as in the original method. The Thompson-Haskell model has been extended by Solyanik [7] to the case of wave propagation through orthotropic layers having differing properties but for which the plane of incidence is always a plane of symmetry. Because of the symmetry of this case, the transfer matrix is $4 \times 4$, as for isotropic layers. Gilbert and Backus [8] and later Richards [9] rewrote the Thompson-Haskell algorithm for the isotropic case in a different form considering the boundary vector as a solution of a system of differential equations

$$
\frac{\partial U}{\partial Z}+i k A U=0
$$

where the transfer matrix $B$ of equation (1) now has the form

$$
B=e^{-i k A h}
$$

Eric Adler and his coworkers at McGill University [10-12] generalized the Gilbert-Backus [8] algorithm to the case of arbitrary anisotropic piezoelectric layered media. In their treatment, the vector $U$ and equation (4) are written in an 8-dimensional space and they found algorithms to calculate the $8 \times 8$ matrices $A$ of equation (4) and $B$ of equation (5).

\section{Elastic wave interaction with layered composites and determination of elastic con- stants}

One of the important requirements for advanced composite materials is that the stiffness satisfy the levels preset by the design. Such a requirement underlines the importance of nondestructive evaluation as a quality control tool to establish that the elastic properties of a manufactured composite are equivalent to those of the designed composite. Several recent papers have been devoted to development of such nondestructive techniques, mainly based on application of bulk ultrasonic waves [13-18] and Lamb waves [19-20].

Here we discuss wave interaction with composite plates when the wavelength is comparable to the thickness. We will also consider a nonlinear inversion scheme to reconstruct the full matrix of elastic constants from ultrasonic experimental data on the reflection from an orthotropic composite 
plate immersed in a fluid.

Ultrasonic wave interaction with a unidirectional composite plate immersed in a fluid has been considered by Nayfeh and Chimenti [21]. They extended their solution to a general laminate [23,24] which generalizes the Thompson [1] matrix method and found expressions for the anisotropic transfer matrix B. An alternative method which is more stable to noise at higher frequency but more computationally intensive has been used by Mal [24].

The coefficient of reflection of ultrasonic waves from a solid plate immersed in a fluid has irregular oscillatory behavior as a function of incident angle and frequency. The maxima of the reflection coefficient alternate with the minima which correspond to real or complex zeroes. The reflection coefficient minima are usually represented in the form of a family of dispersion curves with incident angle $\theta$ (or projection of the wave velocity on plate plane $\mathrm{V}_{0} / \sin \theta$ ) on the $y$ axis and parameter $f h$ on the $x$ axis ( $f$ is frequency and $h$ is plate thickness). Experimentally these curves may be obtained by changing the frequency at a given angle of incidence and finding reflection coefficient zeroes or by changing the angle of incidence at a given frequency. The zeroes of the reflection coefficient have been for a long time closely associated with the Lamb wave spectrum in the plate, which is not always correct. The actual relation between reflection coefficient minima and the spectrum of leaky Lamb waves, which are poles of the reflection coefficient, has been studied in detail only recently [25].

Intensive theoretical and experimental studies of the zeroes of the reflection coefficient spectrum for a composite material have been reported [21,22]. The reflection coefficient $R$ and transmission coefficient $\mathrm{T}$ may be written in the following general form [21]:

$$
R=\frac{A S-Y^{2}}{(S+i Y)(A-i Y)} ; \quad T=\frac{i Y(S+A)}{(S+i Y)(A-i Y)}
$$

where $A$ and $S$ are the symmetric $(S)$ and antisymmetric $(A)$ Lamb wave dispersion functions for an orthotropic plate in a vacuum and $(S+i Y)$ and $(A-i Y)$ are those for a plate in a fluid (dispersion functions of leaky Lamb waves). The function $Y$ appears due to fluid loading and depends on fluid parameters.

The solution of the inverse problem - to find the material properties $\left(C_{i n}, \rho\right)$ of a composite, from experimental data on RC minima (in the $f h-\theta$ plane each experimental point " $i$ " of the $\mathrm{RC}$ minima is determined by measured values of parameters $\theta_{i}$ and $\left(f h_{i}\right)$ - has been reported by Rokhlin and Chimenti [26]. An alternative approach has been discussed by Karim et al [27].

Fig. 1 shows experimental and calculated minima of the reflection coefficient from a unidirectional composite plate [26] for different angles of fiber orientation $\phi$. The elastic constants used for calculation have been reconstructed from the same set of experimental data. The fit is excellent. During this study we found it difficult to reconstruct the longitudinal elastic constant $C_{11}$ in the fiber direction. Experimental data for very small angles of incidence are needed for this.

The alternative approach is to use experimental data on zeroes of the transmission coefficient (Rokhlin et al [28]). This method is extremely sensitive to the longitudinal and shear properties of the composite plate. The transmission coefficient was convenient to use in equation (6).

As one can see from equation (6) the zeroes of the transmission coefficient (reflection maxima) will occur when

$$
S+A=0
$$

The difference between the zeroes of the transmission coefficient (Eq. (7)) and the zeroes of the reflection coefficient which are solutions of the equation:

$$
A S-Y^{2}=0
$$

The solutions for the zeroes of the transmission coefficient are independent of the fluid properties (the function $Y$ ) and in general are different from the roots of the functions $S$ or $A$ (from wave numbers for Lamb waves in the plate). Physically the zero of the transmission coefficient (full isolation) occurs when, due to the interaction of different vibrations in the plate, the normal displacement on the back of the plate vanishes due to compensation of the symmetric and antisymmetric components of the displacement. In contrast, the solution of equation (8) depends on fluid loading [25]. When the fluid density is small the effect of the function $Y$ in equation (8) is negligible and zeroes of the reflection coefficient occur at incident angles close to those for Lamb wave excitation.

The experimental and theoretical results for transmission minima [28] are shown in Fig 2. The calculations have been done using the set of elastic constants reconstructed from the data shown. It has been shown that the inversion procedure has advantages for reconstruction of longitudinal and shear properties compared to use of reflection minima spectra [26]. 

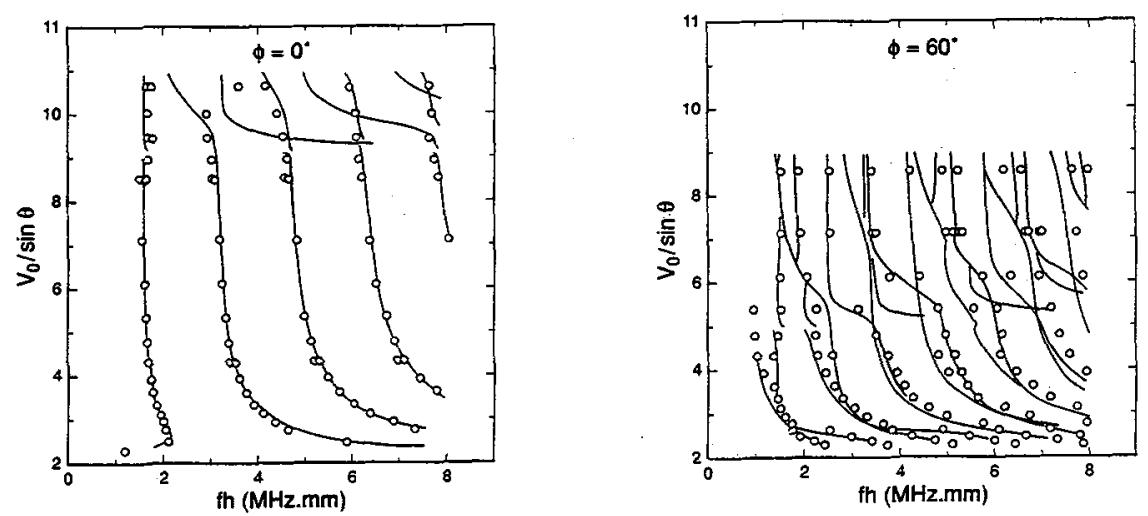

Figure 1. Loci of the reflection minima calculated from reconstructed elastic constants.
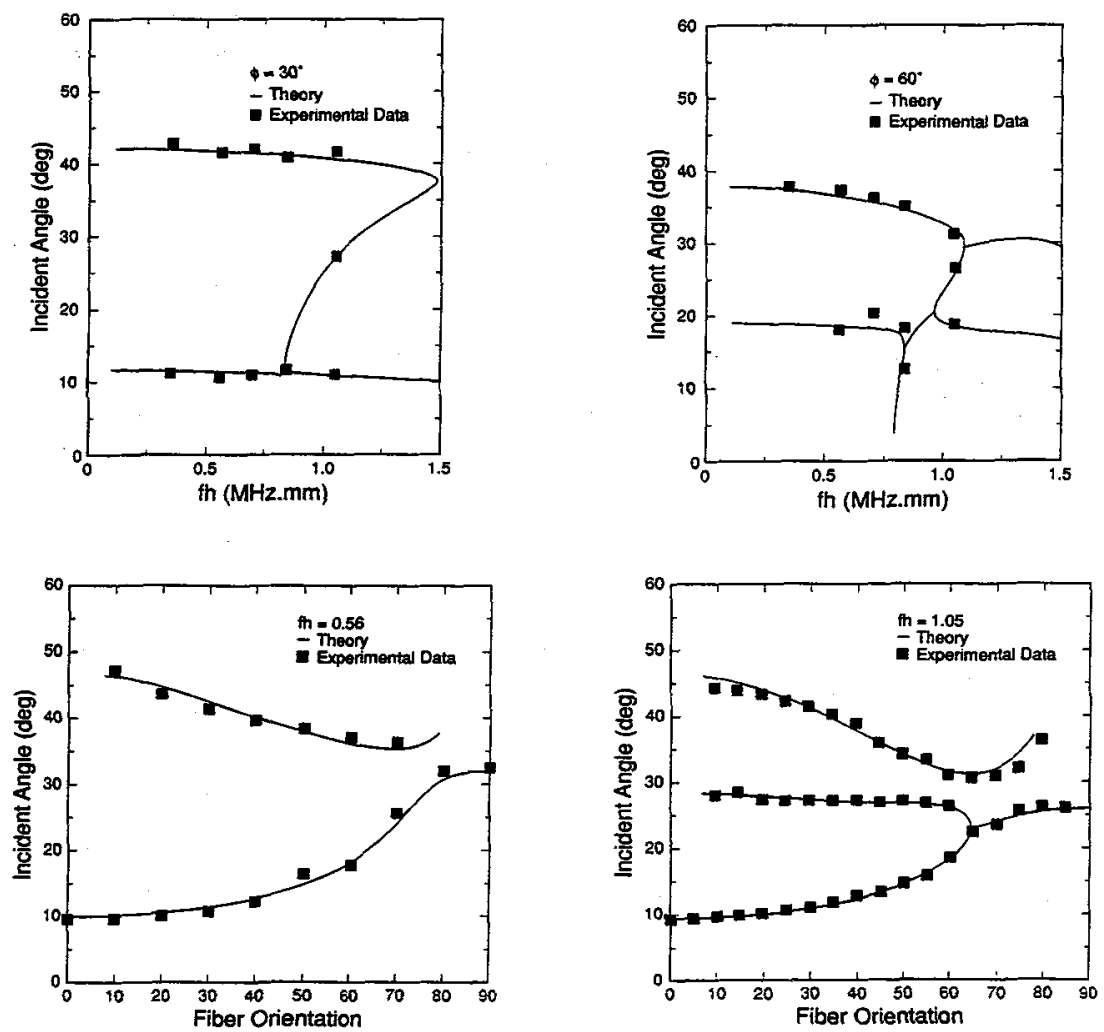

Figure 2. Theoretical dependence of the transmission minima versus parameter $f h$ (top row) and fiber orientation angle (bottom) calculated from reconstructed elastic constants. 


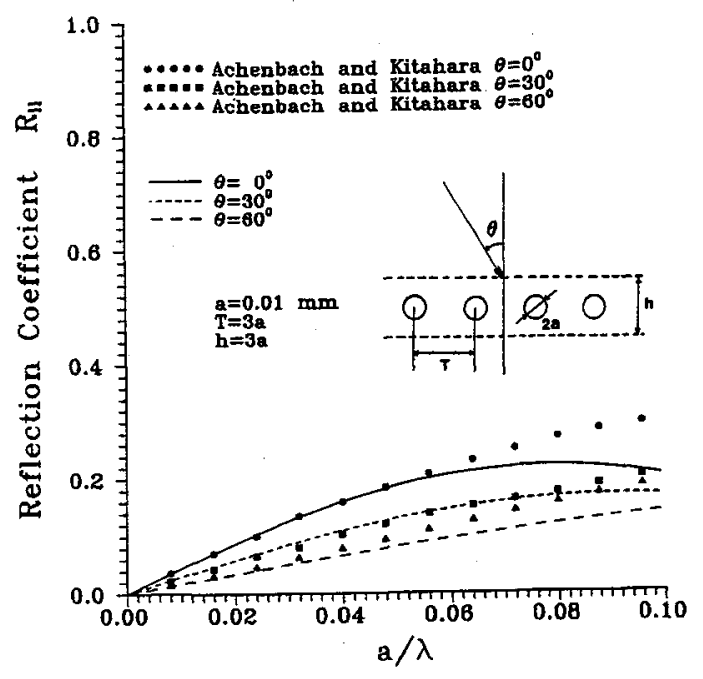

(a)

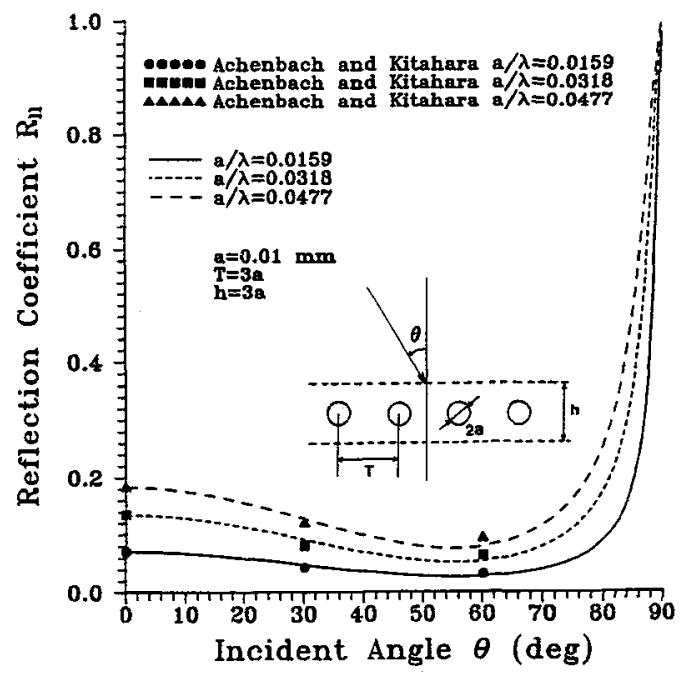

(b)

Figure 3. Reflection coefficient from an array of voids (a) versus radius/wavelength ratio at different angles of incidence; (b) versus incident angle.

\section{Interaction with imperfect interface}

There are two classical boundary conditions for solid/solid interfaces. One boundary condition is for the welded or perfectly bonded interface, which implies continuity of stress and displacement across the interface. The other is the slip boundary condition, which implies continuity of the normal components of stress and displacement across the interface, vanishing of the shear component of stress and discontinuity of the shear component of displacement.

An actual interface between two solids is much more complicated and has physical properties different from those of the substrates. For example even grain boundaries in polycrystalline materials are not perfect because of misfit of the atomic structures of two neighboring grains. In this case dislocations may form on the interface and the atomic structure may become different than in the bulk medium. This interface imperfection may be felt only at very high frequencies. Another example of formation of a thin interface layer occurs when two solids are bonded together either by a thin layer of another material, e.g. glue, or by some metallurgical process.

Rokhlin and Wang [29] consider a model of an imperfect interface with the microdefects as a thin composite layer with averaged effective properties. Fig. 3 illustrate the application of this model to an array of voids in the solid by comparing with the exact numerical solution from references $[30,31]$. We can conclude that a very simple model of the effective composite layer can be used successfully for approximate calculation of the reflection and transmission coefficients through a single row of imperfections on an interface between solids. For multiple rows of imperfections the precision of the model will improve, selection of layer thickness will be obvious and in the limiting case of a large number of rows the model transforms into the standard representation of a composite layer.

\section{Boundary conditions for anisotropic interface}

In some cases an imperfect interface may be modeled as a very thin anisotropic layer, Below we introduce generalized boundary conditions for an anisotropic interface. This is a generalization of our recent work [32] in which we derived approximate boundary conditions for an interface with 
a thin isotropic layer. The boundary conditions are obtained by an asymptotic expansion of the transfer matrix which relates stresses and displacements on opposite sides of the layer.

To derive the reflection and transmission coefficients we use the transfer matrix $[B]$ in form of equation (5), which relates the particle displacement $u_{i}$ and stresses $\sigma_{i k}$ on the top interface to the quantities $u_{i}^{\prime}, \sigma_{i k}^{\prime}$ on the bottom interface of the anisotropic layer:

$$
\left(u_{x}, u_{y}, u_{z}, \sigma_{z x}, \sigma_{z y}, \sigma_{z z}\right)^{T}=B\left(u_{x}^{\prime}, u_{y}^{\prime}, u_{z}^{\prime}, \sigma_{z x}^{\prime}, \sigma_{z y}^{\prime}, \sigma_{z z}\right)^{T}
$$

where the $6 \times 6$ matrix $A$ in equation (5) takes the form (ref. [10-12]). Here $k$ is the component of the wave vector parallel to the interface and $h$ is the thickness of the layer. For a thin interface layer (small $\mathrm{h}$ ), we will limit ourselves to the first terms in an matrix series expansion,

$$
B \approx I-i k h A
$$

Substituting this approximation into equation (9), we obtain the approximate boundary conditions which relate the elastic field on one side of the layer to that on the other side. For a thin orthotropic layer of arbitrary orientation or monoclinic material between two solids, the asymptotic transfer matrix $[B]$ has been obtained by us in the form [33-34]:

$$
[B]=\left[\begin{array}{cccccc}
1 & 0 & b_{13} & \frac{1}{K_{n 1}} & b_{15} & 0 \\
0 & 1 & 0 & b_{15} & \frac{1}{K_{n 2}} & 0 \\
\frac{C_{13}}{C_{33}} b_{13} & \frac{C_{36}}{C_{33}} b_{13} & 1 & 0 & 0 & \frac{1}{K_{n 3}} \\
-\omega^{2} M_{p 1} & b_{51} & 0 & 1 & 0 & \frac{C_{13}}{C_{33}} b_{13} \\
b_{51} & -\omega^{2} M_{p 2} & 0 & 0 & 1 & \frac{C_{36}}{C_{33}} b_{13} \\
0 & 0 & -\omega^{2} M_{n} & b_{13} & 0 & 1
\end{array}\right]
$$

As we can see, coupling terms $b_{15}, b_{51}$ and $b_{13}$ and inertial terms $M_{n}, M_{p 1}$ and $M_{p 2}$ are involved in the boundary conditions. $b_{15}$ and $b_{51}$ couple the in-plane component of displacement to the out-of-plane component of stress or vice versa and the terms involving $b_{13}$ couple the normal component of stress or displacement to the shear component of stress and displacement respectively. The mass factors $q_{1}$ and $q_{2}$ manifest the factors of differences between the inertial terms $M_{p 1}, M_{p 2}$ and $M_{n}$. They equal one at normal incidence, and decrease as the incident angle increases. The $q$ factors depend on the layer properties, and it is not unusual for these factors to become negative at high angles of incidence [35]. The reader is referred to [32,35-36] for a detailed discussion of the behaviors of the corresponding terms for the case of an orthotropic layer with a plane of symmetry coinciding with the incident plane, or of an isotropic layer.

The transfer matrix may be simplified by neglecting coupling terms for materials with little anisotropy, and further simplified by neglecting inertial terms for low density materials.

\section{Interface waves along a thin anisotropic interface layer}

In this section we will briefly discuss the characteristic equations for interface waves and compare results for interface wave velocity from different approximations. This generalizes our previous results for thin isotropic layers [37,38] and orthotropic layers with a plane of symmetry coinciding with the incident plane $[35,36]$ to the anisotropic case . Measuring the velocity of the interface wave localized in a thin anisotropic layer between two solids can be used to evaluate the effective elastic properties of this layer. The characteristic equation for such interface waves is obtained by setting the determinant of the boundary condition matrix to zero.

As an example, let us consider identical aluminum semispaces with the parameters $V_{l}=$ $6.20 \mathrm{~km} / \mathrm{s}, V_{t}=3.24 \mathrm{~km} / \mathrm{s}, \rho=2.7 \mathrm{~g} / \mathrm{cm}^{3}$. The interface layer material is aluminum filled with cylindrical pores oriented parallel to the interface. Let $\varphi$ denote the deviation angle between the pores direction and the interface wave direction. Christensen's model was used to calculate the effective elastic constants of the interface layer. The interface wave velocity divided by the shear 


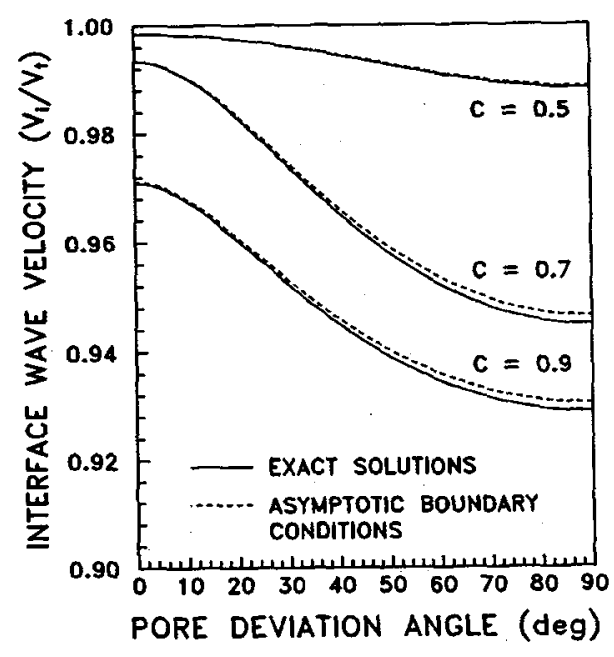

(a)

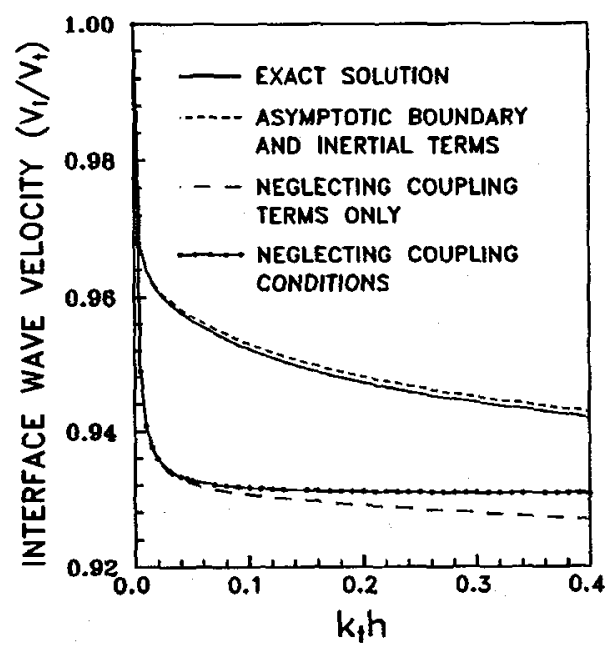

(b)

Figure 4. (a)Exact and asymptotic solutions for interface wave velocity as function of pore orientation $\left(k_{t} h=0.1\right)$. (b) Normalized interface wave velocity for the exact solution and for different approximations of boundary conditions as function of parameter $k_{t} h$.

wave velocity of the substrates is plotted in Fig. 4(a) as a function of pore deviation angle $\varphi$ for $k_{t} h=0.1\left(k_{t}=\omega / V_{t}\right)$ at different pore concentrations. This figure shows very good agreement between the solution from the asymptotic boundary conditions (the dashed line) and the solution from the exact equation (the solid line).

The calculations of the normalized interface wave velocities are compared for different approximate boundary conditions in Fig. $4(\mathrm{~b})$ as a function of the nondimensional interface layer thickness $k_{t} h$ at $\varphi=30^{\circ}$ and pore concentration $C=0.9$. The solutions using boundary conditions which neglect either coupling terms only, or both coupling terms and inertial terms, are quite different from the exact solutions even for small $k_{t} h$, while the solution using the asymptotic boundary conditions shows good agreement for $k_{t} h$ up to 0.4 . This shows the necessity of including all the coupling and inertial terms when analyzing interface wave phenomena.

\section{REFERENCES}

/1/ Thompson, W. T., J. Appl. Phys., 21 (1950), 89-93.

12) Haskell, N., Bull. Seism. Soc. Am., $\underline{43}$ (1953), 17-34.

/3/ Brekhovskikh, L. M., "Waves in Layered Media", Second ed., Academic Press, New York (1980).

/4/ Dunkin, J. W., Bull. Seism. Soc. Am., $\underline{55}$ (1965), 335-338.

/5/ Thrower, E. N., J. Sound Vib., 2 (1965), 210-226.

16/ Kennett, B. L. N., "Seismic Waves Propagation in Stratified Media", Cambridge Univ. Press, Cambridge, England (1983).

17) Solyanik, F. I, Sov. Phys. Acoust., 23 (1977), 533-536.

18/ Gilbert, F. and Backus, G. E., Geophysics, 31 (1966), 326-332.

19/ Richards, P. G., Geophysics, $\underline{36}$ (1971), 798-809.

/10/ Fahmy, A. M. and Adler, E. L., Appl. Phys. Lett., 22 (1973), 495-497.

/11/ Fahmy, A. M. and Adler, E. L., Proc. IEEE, 122 (1975), 470-472. 
/12/ Adler, E. L., IEEE Tranc. Ultrason. Ferroelec. Freq. Contr., 37 (1990), 485-490.

/13/ Hosten, B., Deschamps, M. and Tittman, B. R., J. Acoust. Soc. Am., underline82 (1987), 1763-1770.

/14/ Pearson, L. H. and Murri, W. J., in Review of Progress in Quantitative NDE, ed. by Thompson, D. O. and Chimenti, D. E. (Plenum Press, New York, 1987), Vol. 6B, 1093-1101.

/15/ Kline, R. A. and Chen, Z. T., Materials Evaluation $\underline{46}$ (1988), 986-992.

/16/ Rokhlin, S. I. and Wang, W., in Review of Progress in Quantitative NDE, ed. by Thompson, D. O. and Chimenti, D. E. (Plenum Press, New York, 1989), Vol. 8B, 1489-1496.

/17/ Rokhlin, S. I. and Wang, W., J. Acoust. Soc. Am. 84(1989), 1876-1882.

/18/ Rokblin, S. I. and Wang, W., J. Acoust. Soc. Am. submitted.

/19/ Rose, W. R., Rokhlin, S. I. and Adler, L., in Review of Progress in Quantitative NDE, ed. by Thompson, D. O. and Chimenti, D. E. (Plenum Press, New York, 1987), Vol. 6B, 1111-1118.

/20/ Tang, B. and Henneke II, E. G., Res. Nondestr. Eval. 1 (1989), 51-64.

121/ Nayfeh, A. H. and Chimenti, D. E., J. Appl. Mech. $\underline{55}$ (1988), 863-870.

/22/ Nayfeh, A. H. and Taylor, T. W. and Chimenti, D. E., in Proc. ASME Symp. on Wave Propagation in Structural Composites, Mal, A. K. and Ting, T. C. T., (eds.), ASME, Vol. 9A (1988), 17-27.

/23/ Nayfeh, A. H. and Chimenti, D. E., J. Acoust. Soc. Am. 89 (1991), 542-549.

/24/ Mal, A. K., Wave Motion, 10 (1988), 257-266.

/25/ Chimenti, D. E. and Rokhlin, S. I., J. Acoust. Soc. Am. 88 (1990), 1603-1611.

/26/ Rokhlin, S. I. and Chimenti, D. E. in Review of Progress in Quantitative NDE, ed. by Thompson, D. O. and Chimenti, D. E. (Plenum Press, New York, 1990), Vol. 9B, 1411-1418.

/27/ Karim, M. R. and Mal, A. K. and Bar-Cohen, Y in Review of Progress in Quantitative NDE, ed. by Thompson, D. O. and Chimenti, D. E. (Plenum Press, New York, 1990), Vol. $9 \mathrm{~A}, 109-116$.

/28/ Rokhlin, S. I., Wu, C. Y. and Wang, L. in Review of Progress in Quantitative NDE, ed. by Thompson, D. O. and Chimenti, D. E. (Plenum Press, New York, 1990), Vol. 9B, 1403-1410.

/29/ Rokhlin, S. I. and Wang, Y. J. in Review of Progress in Quantitative NDE, ed. by Thompson, D. O. and Chimenti, D. E. (Plenum Press, New York, 1991), Vol. 10A, 185-192.

/30/ Achenbach, J. D. and Kitahara, M., J. Acoust. Soc. Am. $\underline{80}$ (1986), 1209-1214.

/31/ Achenbach, J. D. and Xu, Y, Ultrasonics Symposium Proceedings (1989, 1107-1111.

/32/ Rokhlin, S. I. and Wang, Y. J., J. Acoust. Soc. Am., $\underline{89}$ (1991), p. 503-515.

/33/ Rokhlin, S. I. and Huang, W., in Review of Progress in Quantitative NDE, ed. by Thompson, D. O. and Chimenti, D. E. (Plenum Press, New York, 1992), submitted

/34/ Rokhlin, S. I. and Huang, W., J. Acoust. Soc. Am., submitted.

/35/ Wang, Y. J. and Rokhlin, S. I., J. Acoust. Soc. Am., (accepted).

/36/ Rokhlin, S. I. and Wang, Y. J., in Review of Progress in QNDE, edited by Thompson, D. O. and Chimenti, D. E. (Plenum Press New York, 1991), Vol. 10A, p. $185-192$

/37/ Rokhlin, S. I., Hefets, M. and Rosen, M., J. Appl. Phys., 51 (1980), p. 3579-3582.

/38/ Rokhlin, S. I., Hefets, M. and Rosen, M., J. Appl. Phys., $\underline{52}$ (1981), p. 2847-2851. 